\title{
MIGRATING SCLERAL EXPLANTS
}

\author{
LUMINA P. LANIGAN, NICHOLAS WILSON-HOLT, ZDENEK J. GREGOR \\ London
}

\begin{abstract}
SUMMARY
Anterior migration of an extrascleral explant is an uncommon complication following buckling procedures. We report five patients where the explant cheesewired through the rectus muscle insertion following retinal detachment surgery. Muscles were not disinserted at the time of surgery in any case. Ocular motility problems were only seen in two patients. The probable mechanisms of this hitherto unreported complication are discussed.

The first recorded scleral buckling operation was performed almost 60 years ago ${ }^{1}$; it involved placing an unsutured surgical sponge between Tenon's capsule and the sclera, with the intention of subsequently removing it. There have since been many improvements in the material and design of scleral explants. ${ }^{2,3}$ They are now better tolerated and it is now common practice to suture the explant to the sclera. Occasionally scleral explants have to be removed if they protrude through the conjunctiva, if they become uncomfortable or infected and if they cause recurrent subconjunctival haemorrhages or extraocular motility dysfunction. ${ }^{4,5,6}$ More serious late complications such as erosion through the sclera under the choroid and/or the retina are now rare. In this paper we report the anterior migration of scleral explants in five patients, in each case apparently cheesewiring through the rectus muscle insertion under which each buckle had been sutured to the sclera. To the best of our knowledge this is the first series of patients to be reported with this complication.
\end{abstract}

\section{PATIENTS AND METHODS}

\section{(1) Surgical Procedure:}

The same surgical technique was adopted in the five cases reported in this series and this involved a $360^{\circ}$ conjunctival peritomy with traction sutures passed under the muscle insertions. The muscles were not disinserted in any case. We used 5.0 braided polyester box sutures with (a) partial thickness posterior circumferential bites and (b) anterior radial bites through the muscle ring at the level of the muscle insertions. The anterior and posterior bites of

From Moorfields Eye Hospital, City Road, London EC1V 2PD.

Correspondence to: Zdenek J. Gregor FRCS FCOphth, Moorfields Eye Hospital, London EC1V 2PD. these sutures were spaced apart by a distance equal to the width of the buckle plus $3 \mathrm{~mm}$. Sutures were placed on either side of muscle insertions and secured under tension, giving rise to a scleral indentation along both the anterior and posterior edges of the explant.

\section{(2) Case Reports}

Case 1. A 53-year-old male underwent right retinal detachment surgery in 1986: a circumferential solid silicone explant was secured under the medial rectus insertion. Postoperatively he achieved $6 / 6$ vision and his retina was reattached. Four years later he complained of ache and irritation in this eye. Examination revealed an exposed explant encroaching onto the limbus. The explant had apparently cheesewired through the medial rectus insertion (Fig. 1). The anterior part of the scleral sutures were not visible. The explant was removed under local anaesthesia. At all times he had a full range of extraocular movement and no diplopia. Postoperatively he had prominent episcleral anterior ciliary collaterals from the inferior rectus (Fig. 2a) and superior rectus muscle circulations (Fig. 2b), apparently anastomosing with the medial rectus circulation. An anterior segment flourescein angiogram showed abrupt interruption of the anterior ciliary artery circulation just anterior to the medial rectus muscle insertion (Fig. 3). A high magnification of the explant removed from this case was compared with a new buckle of similar type (Fig. 4). Irregularities were seen on the superior surface and edges of the old buckle.

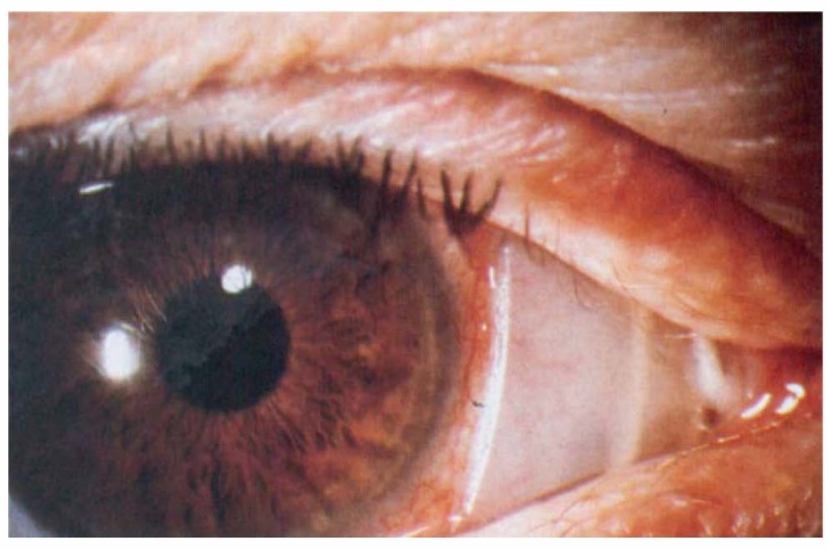

Fig. 1. The exposed anteriorly migrated explant. 


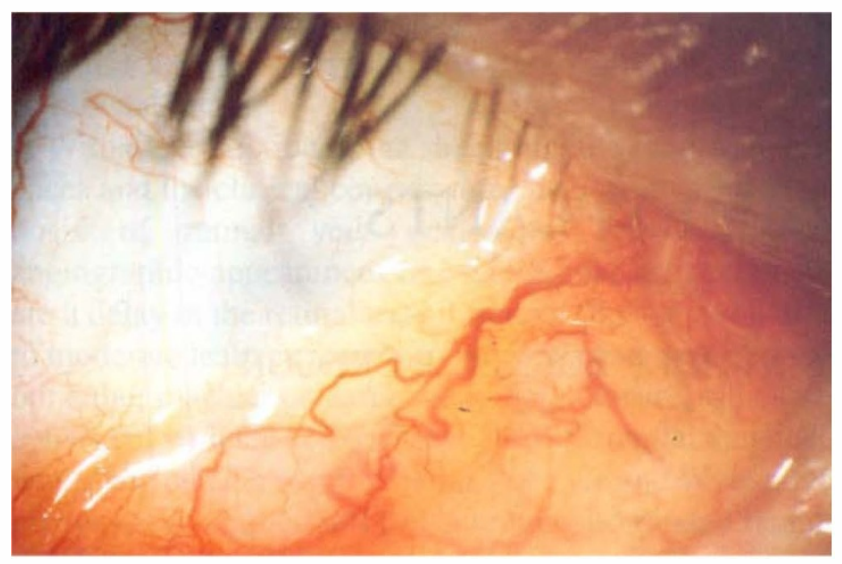

Fig. 2a. Anterior ciliary artery collaterals from the blood supply to the inferior rectus muscle.

Case 2. A 65-year-old male underwent left vitrectomy and an injection of intraocular gas with a solid silicone circumferential explant secured under the superior rectus muscle insertion in February 1988. His retina was successfully reattached and he achieved $6 / 6$ vision in this eye postoperatively. In May 1989 his left visual acuity deteriorated to $6 / 18$ due to cystoid macular oedema and at that examination the superior explant, covered with conjunctiva, was noted to have imigrated anteriorly. The scleral sutures were not visible and were presumed to have cut out. As he was asymptomatic the explant was left alone. In August 1990 he presented with a superior bulbar subconjunctival haemorrhage and on questioning admitted to vertical diplopia when tired. The explant had eroded through the superior rectus insertion and conjunctiva. He had limited elevation of the left eye. The exposed explant was therefore removed in September 1990. By January 1991 his persistent diplopia was relieved with fresnel prisms. Prominent episcleral anterior ciliary collateral channels had developed from neighbouring lateral and medial rectus muscle circulation (Fig. 5a, b). His retina remains attached.

Case 3. A 24-year-old highly myopic female underwent right retinal detachment surgery in September 1990, when an encircling band and a solid silicone circumferential

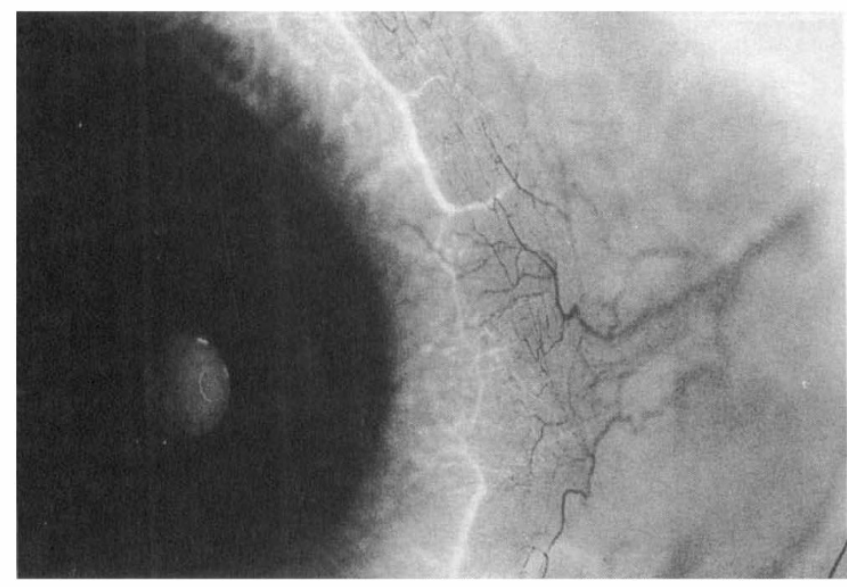

Fig. 3. Interruption of the ciliary artery circulation anterior to the mediall rectus muscle insertion.

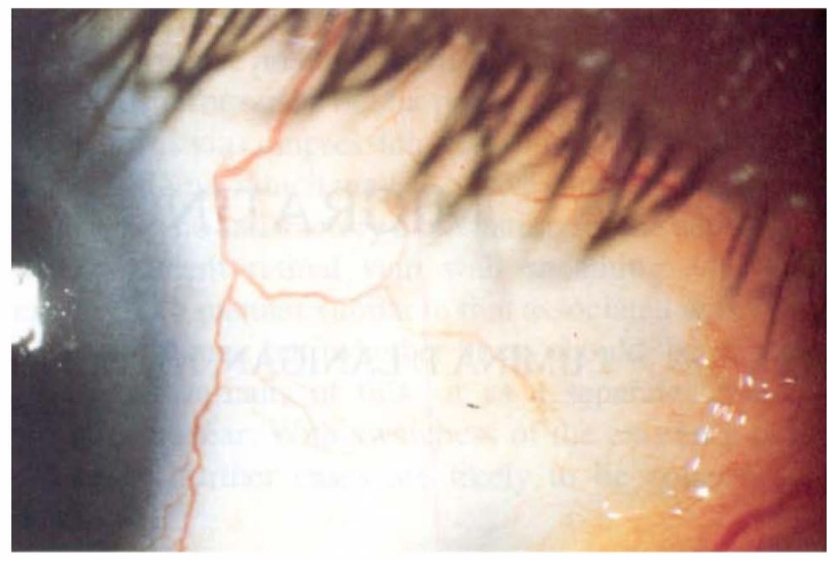

Fig. 2b. Anterior ciliary artery collaterals from the blood supply to the superior rectus muscle.

explant were sutured under the superior rectus muscle insertion. Her retina was reattached and she achieved 6/6 vision postoperatively. She re-presented in February 1991 with a small superior bulbar subconjunctival haemorrhage in the right eye which was managed conservatively. On review, the explant was noted to have migrated anteriorly (Fig. 6a). One month later she re-presented with sudden blurring of vision and a larger subconjunctival haemorrhage in the right eye was noted (Fig. 6b). Slit lamp examination revealed cut out scleral sutures and that the explant, covered with conjunctiva, had migrated anteriorly through the insertion. Her blurred vision was due to a dramatic change in myopic refraction with a change in her post operative astigmatism from $-0.75 \mathrm{DC} \times$ $140^{\circ}$ to $-2.00 \mathrm{DC} \times 40^{\circ}$ which corrected her visual acuity in this eye to $6 / 5$. This astigmatism resolved spontaneously with the absorption of the subconjunctival haemorrhage.

Case 4. An asymptomatic 80-year-old lady at a routine

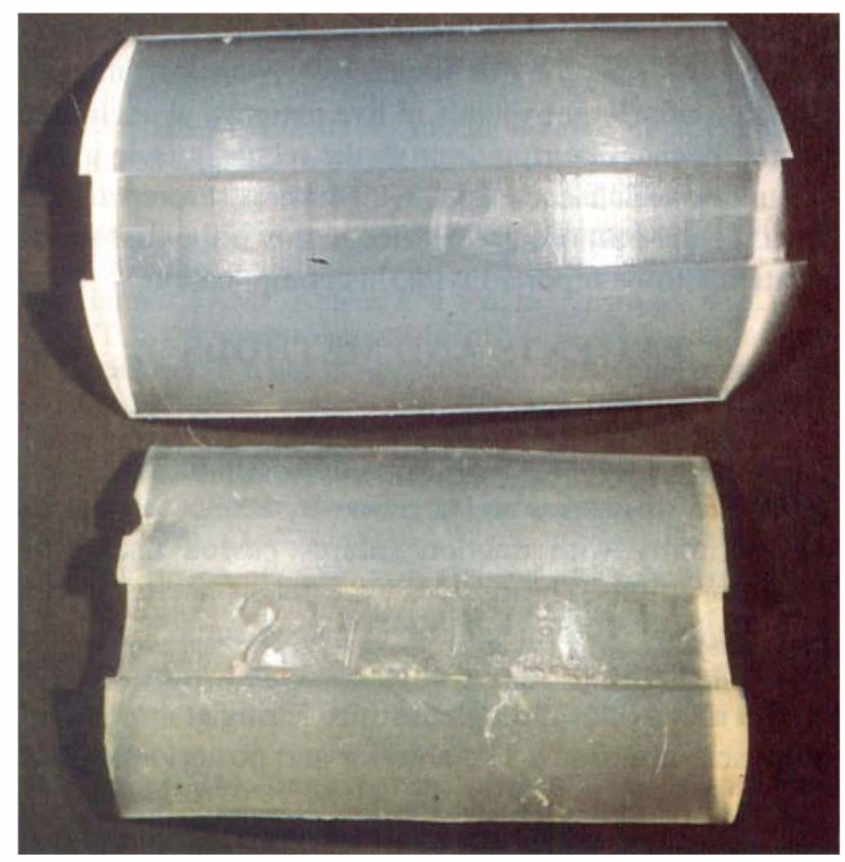

Fig. 4. A comparison of a new explant (above) with the patient's old one (below). 


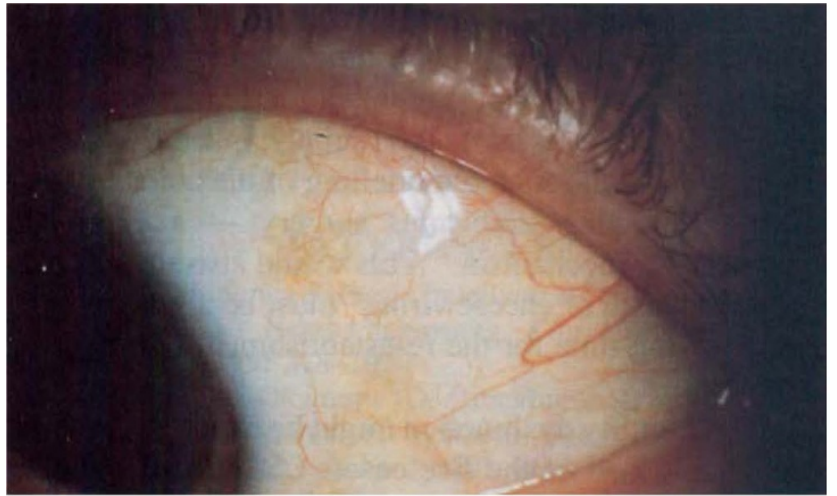

Fig. 5a. Anterior ciliary artery collaterals from the blood supply to lateral rectus muscle.

outpatient appointment was noted to have an exposed anteriorly migrated scleral explant at the superior bulbar area of her only eye (Fig. 7). No scleral sutures were seen on the exposed buckle surface. Five years previously, she had had a right cataract extraction complicated by vitreous loss and a year later developed a retinal detachment. She underwent encirclement with a circumferential solid silicone explant secured under the superior and medial rectus insertions. She required a further operation two weeks later and had the original explant removed and replaced with another secured under the lateral, superior and medial rectus muscle insertions. Postoperatively her retina was reattached but visual acuity remained at count fingers level only. When she was examined by us in November 1990, the explant was exposed and had migrated anteriorly through the superior rectus muscle insertion. She had no diplopia but there was a right hypertropia (by reflections) with restricted right ocular motility in all depressed positions. Forced duction testing at the time of removal of her extruded explant revealed a tight superior rectus. There were anterior ciliary collaterals from the neighbouring recti muscle circulation which became more obvious postoperatively (Fig. 7).

Case 5. A 24-year-old myopic male underwent left retinal detachment surgery in 1982, encirclement with a solid silicone explant secured circumferentially under the lateral rectus muscle insertion between two and four o'clock.

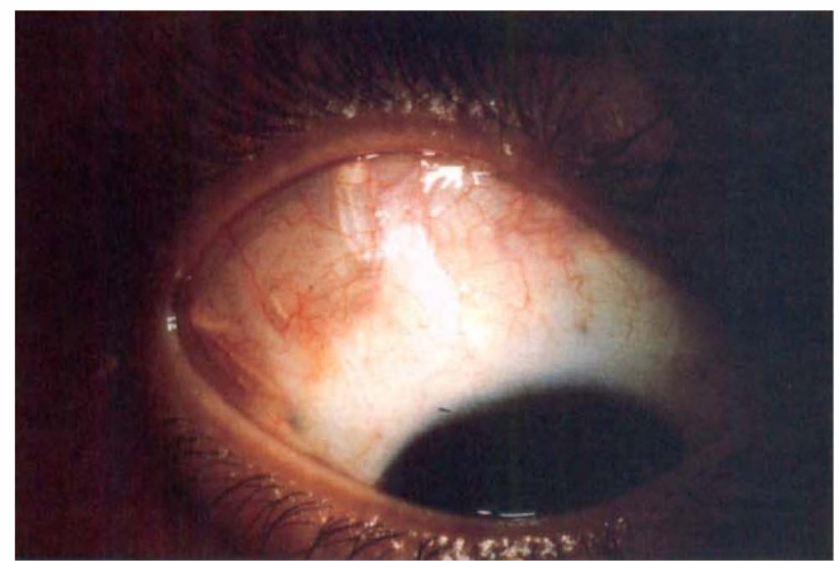

Fig. 6a. The anteriorly migrated superior explant.

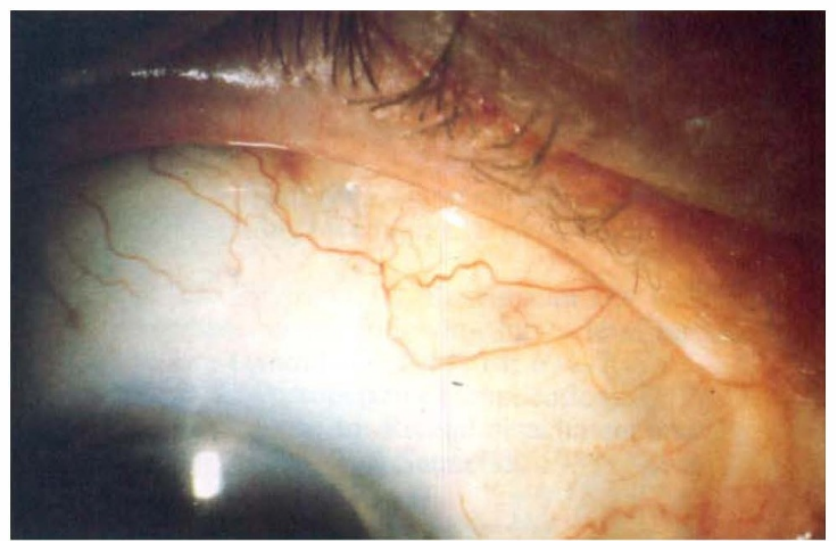

Fig. 5b. Anterior ciliary artery collaterals from the blood supply to the medial rectus muscle.

Postoperatively his retina was reattached and he achieved 6/9 vision. In 1990 he re-presented with a one-week history of ocular discomfort. A large subconjunctival haemorrhage was noted and on slit lamp examination the scleral explant was found to have migrated anteriorly to within $5 \mathrm{~mm}$ behind the scleral limbus, apparently cheesewiring through the lateral rectus muscle insertion but remained covered with conjunctiva (Fig. 8). The scleral sutures had cut out and were visible through the conjunctiva. He had no diplopia but orthoptic assessment revealed slight restriction of adduction and abduction of the left eye. The explant was left alone and the patient remains asymptomatic. By March 1991 prominent episcleral vessels were seen on the lateral bulbar conjunctiva in this eye, suggestive of collateral anastomotic channels between the inferior and superior recti circulations.

\section{DISCUSSION}

These five cases highlight an uncommon complication of extrascleral buckling procedures for retinal detachment operations namely, anterior migration through the rectus muscle insertion. Hamlet et al. reported as case of dehiscence of the lateral rectus muscle but this case followed an intrascleral procedure. ${ }^{7}$ In the present cases inert, solid, silicone explants were secured onto the sclera under one or more rectus muscles. We cannot comment whether this complication can also occur with silicone sponges -

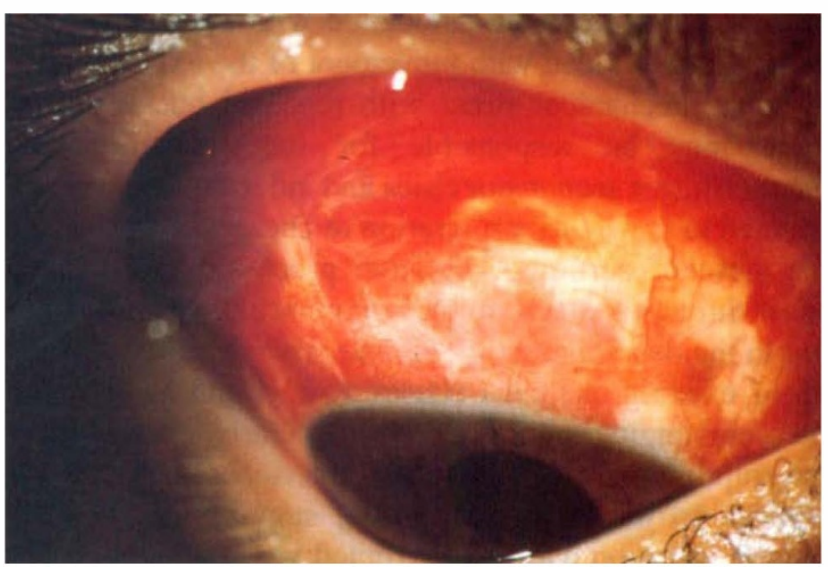

Fig. 6b. Superior subconjunctival haemorrhage. 


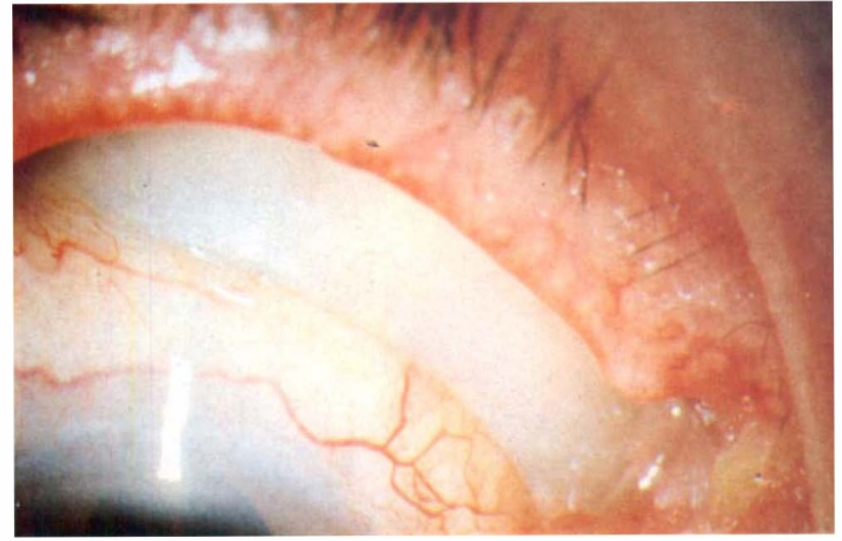

Fig. 7. The exposed anteriorly migrated explant with collaterals from the blood supply to the neighbouring recti muscles.

bearing in mind that they are softer, more pliable and without rigid edges. None of the muscles were temporarily disinserted at the time of retinal detachment surgery. It would appear that none of the recti muscles are immune to this complication as the superior, medial and lateral muscles were affected in this series. There was a variable interval between surgery and the detection of forward migration of the buckle (six months to eight years). In all cases scleral sutures securing the explant in situ at the time of the surgery had either cut out (Cases 2, 3,5) or were not visible (Cases 1, 4 both with exposed explants). The same surgical technique was used in this series. Faulty technique resulting in a circling explant that is too tight, too anteriorly placed and/or insufficiently secured to the sclera may theoretically contribute to erosion of the muscle insertion by the explant (8). Furthermore abnormal/altered muscles may predispose to suture cutout and might therefore contribute to forward migration of the explant. When the explant had extruded through the conjunctiva it was removed under local anaesthesia (cases 2 and 4): in the other three cases the explants were left in situ.

Three cases presented with large subconjunctival haemorrhages mainly related to the position of the explant. Russo suggested that a fibrovascular membrane is formed around the explant and with time forms an easily identified "silicone membrane" with small friable new vessels that are closely associated with the explant and which tend to bleed with movement of the explant. ${ }^{9}$ Progressive mechanical stress with repeated buckle movement may be responsible for the subconjunctival haemorrhages seen in our cases and indeed may have contributed to the anterior migration or cheesewiring through the muscle insertions. The latter process may gradually sever through the anterior ciliary muscular arteries, leading to the development of "collaterals" or rather opening up of existing channels (Cases 1, 2, 4 and 5). The fluorescein angiogram (Case No.1) clearly demonstrates interruption of the episcleral anterior ciliary vessels anterior to the muscle insertion, suggesting a severed muscle insertion and its interrupted vascular supply. The subconjunctival haemorrhage may be part of this phenom- enon. The demonstration of large "collaterals" seen in Cases 1, 2, 4 and 5- suggests that remodelling of the episcleral circulation occurs with time, creating "collateral channels" from neighbouring intact recti muscle circulation to re-establish its own blood supply; indeed Lee et al., have demonstrated such remodelling of the collateral circulation following strabismus surgery - a long-term compensatory mechanism. ${ }^{10}$ This would also suggest that the "mechanism of cheesewiring" must be a gradual process, allowing time for the re-establishment of collateral circulation.

Ocular motility dysfunction might be expected, but was seen in only two of the five cases: Case 2 had diplopia which was corrected by prisms, therefore not requiring strabismus surgery; Case 4 had no symptoms due to poor vision in the contralateral eye, but had markedly restricted ocular movements probably related to repeated surgery with extensive buckling and muscle fibrosis. The case reported by Hamlet et $a l^{7}{ }^{7}$ was surgically explored because of marked esotropia; the lateral rectus muscle had been neatly sheared off at its insertion and found to be reattached to the globe behind the explant. It is possible that the lack of ocular muscle dysfunction in three of the present five cases (all of whom had good vision) may have been due to reattachment of the muscle sheath/fibres relatively close behind the explant, but not too far back to interfere with function. We did not consider it appropriate to explore the cheesewired muscles in this series; in a similar case of anterior explant migration where the muscle insertion was explored, it appeared normal (Lee, JP personal communication).

In none of our cases was there evidence of infection which suggests that the anteriorly directed pressure of the advancing edge of the explant may cause local tissue necrosis and/or ischaemia resulting eventually in "cheesewiring" through the muscle insertion. The irregularities on the surface and edges of the explant removed from Case 1 may have contributed to the trauma of adjacent muscle fibres during ocular movement.

These five cases highlight the fact that spontaneous cheesewiring/dehiscence of recti muscle insertions can occur following routine extrasclera buckling procedures. A larger series is needed before one can comment on the

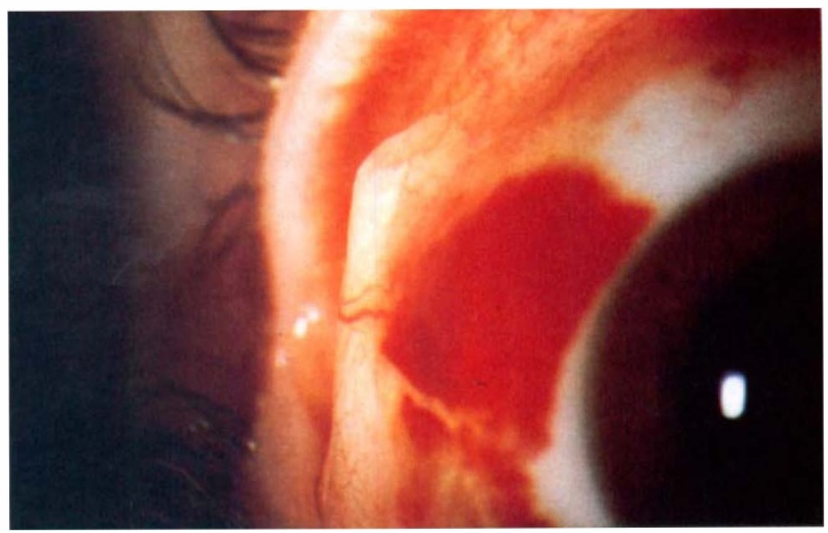

Fig. 8. Subconjunctival haemorrhage with explant migration through the lateral rectus muscle insertion. 
true incidence of ocular motility dysfunction following this complication.

Key words: Scleral explants, Complications, Ocular motility dysfunction.

\section{REFERENCES}

1. Jess A: Temporare Sklerale Indellung als Hillsmittel bei der Operation der Netzhautablosung. Klin Monatsbl Augenheilkd 1937, 99: 318-19.

2. Schepens CL, Okamura ID, Brockhurst RJ et al: Scleral buckling procedures $\mathrm{V}$ : synthetic sutures and silicone implants. Arch Ophthalmol 1960, 64: 868-81.

3. Lincoff HA, Baras I, McLean J: Modifications to the Custodis procedure for retinal datachment. Arch Ophthalmol 1965, 73: 160-3.

4. Roth AM and Sypnicki BA: Motility dysfunction following surgery for retinal detachment. Am Orthopt $J$ 1975, 25: $118-21$.

5. Mets MB, Wendell ME, Gieser RG: Ocular deviation after retinal detachment surgery. Am J Ophthalmol 1985, 99: 667-72.

6. Delaney WV, Tomisi PF, Hampton GR et al: Complications of scleral buckling procedures. Arch Ophthalmol 1987, 105: 703-3.

7. Hamlet YJ, Goldstein JH, Rosenbaum JD: Dehiscence of lateral rectus muscle following intrascleral buckling procedure. Ann Ophthalmol 1982, 14: 694-7.

8. Schepens CL: Postoperative complications: II. Problems of specific procedures. In: Retinal detachment and allied diseases. Philadelphia: WB Saunders, 1983; Vol 2, Chap. 40 1024-28.

9. Russo CE. Ruiz RS: Silicone sponge rejection. Early and late complications in retinal detachment surgery. Arch Ophthalmol 1971, 85: 647-50.

10. Lee JP, and Olver J: Anterior segment ischaemia. Eye 1990, 4: $1-6$. 